\title{
Low-Frequency Temperature Oscillations of Leg Ulcers in Patients with Sickle Cell Anemia
}

\author{
by J. Maivelett*†, A. Koroulakis*†, K. Delaney**, N. Malik*, C.P. Minniti**, W. Liu***, A. Behnaz*, G.J. Kato**, \\ and A.M. Gorbach*
}

*Infrared Imaging \& Thermometry Unit, NIBIB, NIH, Bethesda, MD, USA, gorbacha@mail.nih.gov

* Sickle Cell Vascular Disease Section, Hematology Branch, NHLBI, NIH, Bethesda, MD, USA

${ }_{* \star \star}$ Dept. of Computer Science and Information Engineering, National Chung Cheng University, Taiwan

†These authors contributed equally to this work

\section{Abstract}

Infrared (IR) imaging and Laser Speckle Contrast Imaging ( $\mathrm{LSCl}$ ) were used to measure skin temperature and blood flow, respectively, in leg ulcers of sickle cell anemia patients. Regional maps of the power of oscillations were computed via Fast Fourier Transform within the ulcers and surrounding tissues. We hypothesize that measurements of regional oscillations of temperature and perfusion by IR imaging and $\mathrm{LSCl}$, respectively, may be sensitive, quantitative, non-invasive markers for ulcer pathophysiology, including local vascular tone and functional connectivity between ulcers and surrounding tissues.

\section{Introduction}

Leg ulcers are a common complication of sickle cell disease and often reoccur, possibly due to decreased blood flow at the ulcer site [1]. Clinical data for selecting treatment options are unsatisfactory, as changes in the microcirculation associated with ulceration and healing are often not addressed during wound assessment [1]. Thus, the application of techniques capable of examining microcirculatory changes may have predictive value in the assessment of ulcerated tissue.

IR imaging has been previously shown to be capable of vascular assessment at depths of about $1 \mathrm{~cm}$ [2]. Because blood coming from the body's core is warmer than the exposed skin surface, local blood flow can be used as a natural thermal contrast agent for monitoring of microvascular perfusion, vasomotion, and vascular reactivity [2]. Lowfrequency oscillations of temperature related to changes in vascular tone have previously been assessed [2]. Because the amplitude of heat fluctuations decays as an exponential function of frequency, IR imaging is more suited for measurement of low-frequency temperature fluctuations on the skin surface [2].

$\mathrm{LSCl}$ has been used to measure red blood cell flux directly. LSCl operates by illuminating tissue with a diverging near-infrared laser and imaging the reflected light with a CCD camera. The light is scattered by moving red blood cells, producing a blur in the collected images that is proportional to red blood cell flux through a given region [3]. The LSCI camera calculates flux values by performing a contrast analysis on the image via low-resolution spatial processing, which analyzes variation in intensity within small groups of pixels [4]. Within a given group of pixels, the speckle contrast is denoted by:

$$
\mathrm{K}=\frac{\sigma}{\langle\mathrm{I}\rangle}
$$

where $\langle\mathrm{I}\rangle$ is the mean intensity value and $\sigma$ is the standard deviation. The speckle contrast $\mathrm{K}$ is then converted to arbitrary perfusion units by the following expression:

$$
\operatorname{Flux} \propto\left(\frac{\langle\mathrm{I}\rangle}{\sigma}\right)^{2}
$$

Although the use of LSCI for assessment of skin microvascular function has increased in recent years, the low-frequency oscillations in blood flow associated with changes in vascular tone have yet to be explored.

Oscillations in skin microvasculature below $0.1 \mathrm{~Hz}$ are strongly associated with regulation of regional blood flow. Specifically, blood flow oscillations occurring in the $0.005-0.0095 \mathrm{~Hz}, 0.0095-0.021 \mathrm{~Hz}, 0.021-0.06 \mathrm{~Hz}$, and $0.06-$ $0.20 \mathrm{~Hz}$ frequency ranges have been attributed to endothelium-derived hyperpolarizing factor (EDHF), endothelial release of nitric oxide (NO), sympathetic activity, and myogenic activity, respectively [5].

Vasomotion is involved in the regulation of blood flow through establishment of local vasomotor tone and functional connectivity between smooth muscle and endothelial cells [6]. Moreover, vascular networks have the ability to coordinate vasomotion through intercellular communication [7]. These coordinated oscillations may be distinguishable by IR imaging and LSCI techniques.

Although IR and LSCI techniques have been used previously to examine ulcerated tissue [8-10], low-frequency oscillations of temperature and blood flow related to vascular tone have yet to be assessed. We hypothesize that fluctuations in these parameters could be used as sensitive, non-invasive markers to quantify different aspects of 
vascular pathophysiology in the ulcer bed and surrounding tissues. In this study, we assess microcirculatory changes related to vascular tone in the ulcer and surrounding tissue through the imaging of temperature and blood flow, followed by analysis of their respective oscillations in spatial, temporal, and frequency domains.

\section{Methods}

\subsection{Subjects}

Ten patients with leg ulcers concurrent with sickle-cell anemia were studied under NIH Protocol 11- $\mathrm{H}-0121$. The study group contained 4 males and 6 females with average age $42.0 \pm 10.2$ years, height $173.1 \pm 8.5 \mathrm{~cm}$, and weight $68.4 \pm 9.0 \mathrm{~kg}$. Examined ulcerations were on the medial $(n=5)$ and lateral $(n=5)$ sides of the leg and ranged in surface area from 2.51 to $7.17 \mathrm{~cm}^{2}$. During the time of study, all subjects were in-patients at the NIH Clinical Center.

Eligibility criteria required each subject be at least 18-years-old, have sickle cell anemia or other hemoglobinopathy or another hemolytic disorder (e.g., hereditary spherocytosis), and have a leg ulcer of at least 4 weeks duration and 2.5 to $100 \mathrm{~cm}^{2}$ in surface area. All subjects gave informed consent.

\subsection{Imaging}

Prior to each imaging session, visible light photographs of the ulcer were taken for later use as references when selecting regions of interest (ROIs). Imaging was performed after 20 minutes of acclimatization at a room temperature of $\sim 22^{\circ} \mathrm{C}$ with patients in the supine position. Patients were instructed to minimize leg motion for the duration of imaging. $\mathrm{A}$ calibrated IR camera (Santa Barbara Focalplane Array, Lockheed Martin, USA) with a temperature resolution of $0.015^{\circ} \mathrm{C}$ (3.0-5.0 $\mu \mathrm{m}$ wavelength, $640 \times 512$ pixels/image, 16 bits/pixel) was employed to detect temperature at a sampling rate of $\sim 1.9 \mathrm{~Hz}$. In conjunction with IR thermography, an LSCI camera (model moorFLPI, Moor Instruments Ltd., USA) with flux measurement accuracy of $\pm 10 \%$ was used to observe red blood cell flux at a sampling rate of $\sim 5.0 \mathrm{~Hz}$ (as well as $785 \pm 10 \mathrm{~nm}$ laser wavelength, $152 \times 113$ pixels per image, 8 bits/pixel). IR and LSCI cameras were directed toward the site of ulceration at distances of $\sim 42$ and $\sim 32 \mathrm{~cm}$ from the ulcer center, respectively. Images were collected by both cameras simultaneously during a 25 -min baseline period for each of the ten subjects. The collected image sets ( 2880 images for IR and $\sim 7500$ images for LSCI) provided the capability to extract time series for each pixel.

\subsection{Image Processing}

\subsubsection{Image Alignment}

Image registration was performed using built-in functions of the Statistical Parametric Mapping Toolbox under MATLAB 7.10 (MathWorks, Inc., USA) to reduce motion artifacts. A single image with three recognizable fiducial markers (reflective film) was used as a reference to align the remaining images by smoothing with a 3x3 Gaussian kernel matrix and B-spline resampling, followed by a least-squares rigid body transformation [11].

\subsubsection{ROI Selection}

Selected regions of interest were located at the ulcer center (ROI 1), directly adjacent to the ulcer (ROI 2), and $>5 \mathrm{~cm}$ away from the ulcer (ROI 3). Visible light photographs were used as references in determining ROI location. ROIs 2 and 3 were consistently defined most proximal to the body, with the third region (acting as a control ROI) far removed from the area affected by ulceration. Selected regions were recognizable as squares of $\sim 19 \times 19$ pixels in IR and $\sim 5 \times 5$ pixels in LSCI.

\subsubsection{ROI Analysis}

Average temperature and blood flow images were created by time-averaging each pixel across all frames in the 25-minute image sets. The resulting images were then used to find average temperature and blood flow in each ROI by conducting spatial averages of the pixels within each region.

In preparation for frequency analysis, frequency oscillations below $0.005 \mathrm{~Hz}$ were removed from the raw image sets using a highpass elliptical filter with a cutoff frequency of $0.004 \mathrm{~Hz}$ [12]. Temperature and blood flow oscillations within each ROI were then assessed using a Fast Fourier Transform (FFT) based on the Cooley-Tukey algorithm. The power spectrum for each ROI was calculated using the following expression:

$$
\bar{P}=\frac{\left|F F T\left(p_{i}\right)\right|^{2}}{N}
$$

where $p_{i}$ is the time series of one pixel, $N$ is the number of pixels within a given ROI, and $\bar{P}$ is the averaged power spectrum for said ROI. The power of oscillations within each frequency range of interest was then assessed by analyzing the power density, or area under the curve (AUC), in each specific range, given by: 


$$
\int_{a}^{b} \bar{P} d f
$$

where $a$ and $b$ denote the lower and upper limits of the frequency range of interest.

The above procedure was used to analyze temperature and blood flow oscillations in the EDHF, NO-dependent endothelial, sympathetic, and myogenic frequency ranges in each ROI. Power density in the full $0.005-0.20 \mathrm{~Hz}$ frequency range associated with local regulation was also calculated for each ROI.

\subsubsection{FFT Image Computation}

The aforementioned FFT algorithm was also used to create images showing power density of temperature and blood flow oscillations at each pixel for individual frequency ranges. After removal of very low frequencies $(<0.005 \mathrm{~Hz})$ using the high-pass elliptical filter, FFT was performed on each pixel of the IR and LSCl images. Power density images were then generated by calculating the power density within a specified frequency range at each pixel. The following expression summarizes this process for one pixel:

$$
P=\int_{a}^{b}\left|F F T\left(p_{i}\right)\right|^{2} d f
$$

where $p_{i}$ is the time series at one pixel, and $P$ is the power density for that pixel in the frequency range $a \mathrm{~Hz}$ to $b \mathrm{~Hz}$.

The above procedure was used to create oscillation images of the power density in the full $0.005-0.20 \mathrm{~Hz}$ range. Separate oscillation images for the EDHF, NO-dependent endothelial, sympathetic, and myogenic frequency ranges were also generated.

\subsubsection{Spatial Profiles}

Spatial profiles (pixel value vs. distance) were generated through the centers of ROls 1, 2, and 3 using built-in functions of the Image Processing Toolbox of MATLAB 7.10. Spatial profiles were extracted from the IR average temperature, IR oscillation, LSCI average blood flow, and LSCI oscillation images. These profiles were then averaged across all patients to provide qualitative observations of spatial changes in temperature, blood flow, and their respective oscillatory activity.

\subsubsection{Wavelet Phase Coherence}

Temperature and blood flow oscillations were also analyzed by wavelet phase coherence (WPC) for one patient $(n=1)$ as a preliminary case study. This analysis was applied for comparison of the selected ROIs and for the generation of WPC images, which were used to show regions of high coherence with the ulcer.

\subsubsection{Computation of Wavelet Phase Coherence (WPC) for Selected ROIs}

In preparation for further frequency analysis, a spatial average of the pixels within each ROI was calculated for each image in the data set, resulting in profiles of ROI temperature or blood flow over time (i.e. average ROI signals). Very low frequencies $(<0.005 \mathrm{~Hz}$ ) were then removed using the high pass elliptical filter, and wavelet transform analysis was performed on each average ROI signal using a Morlet mother wavelet. By converting a signal into the timefrequency domain, wavelet transforms enable analysis of frequency components within a signal while achieving time localization [13]. During the analysis, adjustment of the wavelet scale (i.e. window length) determines the frequency components to be analyzed, and the translation of the mother wavelet within the signal localizes these components in time. Thus, different frequencies correspond to wavelet functions of different window lengths, with lower frequencies requiring wider window lengths for calculation of the frequency response. Areas at the beginning and end of the signal where the window length exceeds the number of points (i.e. overhangs the edges of the signal) mark a region called the Cone of Influence (COI). Inside the $\mathrm{COI}$, edge effects reduce the reliability of the calculated wavelet transform and can significantly influence results $[2,5]$. Thus, values inside this region were removed from further analysis.

The output wavelet transform is a matrix of complex values containing amplitude and phase information at each time point $t_{n}$ and for each frequency component $f_{k}$ [5]. To calculate the wavelet phase coherence between two signals, the relative phase difference, $\Delta \emptyset_{k, n}$, is calculated using the instantaneous phases of both signals obtained from their wavelet transforms: $\Delta \emptyset_{k, n}=\emptyset_{2 k, n}-\emptyset_{1 k, n}$. The sine and cosine components of the phase difference are then calculated and averaged in time for the whole length of the signal, giving the phase coherence, $C_{\emptyset}\left(f_{k}\right)$, between the two signals [13]:

$$
C_{\emptyset}\left(f_{k}\right)=\sqrt{\left\langle\cos \left(\Delta \emptyset_{k, n}\right)\right\rangle^{2}+\left\langle\sin \left(\Delta \emptyset_{k, n}\right)\right\rangle^{2}}
$$

The magnitude of the phase coherence has values between 0 and 1 and expresses the tendency of the phase difference between the signals to remain fixed over time at a given frequency $f_{k}$. A value close to 1 indicates high coherence at the selected frequency $[5,13]$. This procedure was used to calculate the WPC between each average ROI signal. 
To determine significant coherence between signals, a white noise reference curve was created by first generating 30 white noise signals each consisting of 3000 points. These signals were then analyzed using the above procedure for wavelet phase coherence, and the output WPC functions were averaged to generate a mean white noise coherence curve. The threshold for significance was set to one standard deviation above this average white noise coherence curve.

\subsubsection{WPC Image Computation}

Wavelet phase coherence analysis was also used to generate images which compare the oscillations of each pixel to the oscillations of average temperature or blood flow within the ulcer. First, the ulcer was manually traced on IR and LSCI images using built-in functions of the Image Processing Toolbox of MATLAB 7.10. A spatial average of the pixels within this tracing was then calculated for each image in the data set, resulting in a profile of ulcer temperature or blood flow over time (i.e. average ulcer signal). Frequencies below $0.005 \mathrm{~Hz}$ were removed from this signal, and wavelet transform analysis was performed using a Morlet mother wavelet. The resulting ulcer wavelet transform was the consistent element for all upcoming comparisons. The same wavelet transform analysis was performed for the signal in each pixel, and the ulcer and pixel wavelet transforms were compared using wavelet phase coherence. The COI region was excluded during these calculations. Different frequency ranges were then assessed by averaging the phase coherence function across each range. This process was repeated for each pixel in a given image set, resulting in images which replace original pixel value with the average WPC between the ulcer and said pixel across a specified frequency range. The following expression summarizes this process for one pixel in a given range:

$$
P_{W P C}=\frac{\sum_{f_{k} \in S} C_{\varnothing}\left(f_{k}\right)}{X}
$$

where $C_{\varnothing}\left(f_{k}\right)$ is the phase coherence between the pixel and the ulcer, $S$ is the set containing the discrete points of $f_{k}$ in the specified frequency range, $X$ is the number of elements in the set $S$, and $P_{W P C}$ is the average WPC for that pixel in said frequency range.

The above procedure was used to create WPC images for the EDHF, NO-dependent endothelial, sympathetic, and myogenic frequency ranges. Binary images were also created that show pixels with average WPC values above the average white noise threshold for a given frequency range. The white noise coherence curve was calculated by the same procedure used in Section 2.3.6.1 above, and the threshold for significance was considered one standard deviation above this mean curve. The average white noise threshold for a given frequency range was calculated using a procedure similar to equation (7) above.

\subsection{Statistical Analysis}

Non-parametric tests were used to detect differences between ROIs on the basis of average ROI temperature, average ROI blood flow, and average ROI oscillations. ROI oscillations were analyzed in four separate sub-ranges corresponding to different physiological activities by examining the power density (or AUC) within each range. The power density in the full $0.005-0.20 \mathrm{~Hz}$ range was also assessed. A Kruskal-Wallis one-way analysis of variance (ANOVA) on ranks with a Tukey post hoc test for pairwise comparisons was used to determine statistical significance of evaluated parameters across ROIs. A Wilcoxon signed rank test was also used to compare each ROI in a paired fashion. Data for average ROI temperature, average ROI blood flow, and average ROI oscillations were not normally distributed and are therefore expressed as median values across patients.

All statistical analyses were performed using the SigmaStat ${ }^{\circledR}$ 3.5.0.54 software package (Systat Software, Inc., USA).

\section{Results}

Figure 1 shows IR and LSCI images for a representative subject, with visible light photograph and selected ROIs shown for reference. Figure $1 \mathrm{~b}$ shows the average IR image, with temperature values averaged over the 25 minute imaging session for each pixel. Average temperature of the ulcer was visibly lower than the surrounding tissue. Temperature also appeared heightened in the area surrounding the ulcer tissue. Figure 1c shows blood flow of the ulcer and surrounding tissue averaged over 25 minutes. Blood flow was highest within the ulcer and was also increased in the adjacent tissue. Blood flow substantially decreased with distance from the ulcer.

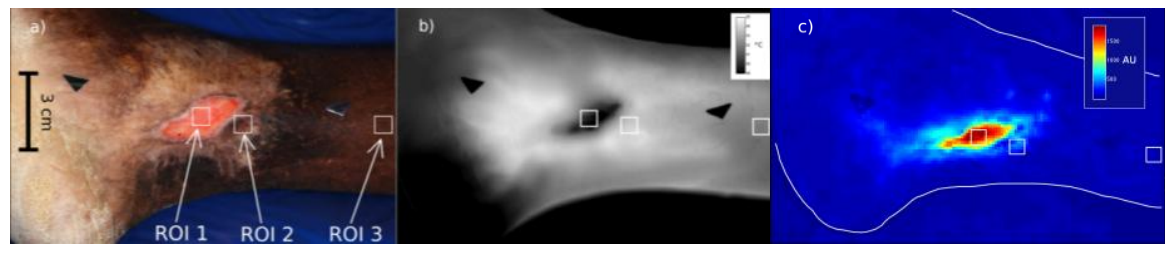

Fig. 1. Representative visible light (a), time-averaged IR (b), and time-averaged $L S C I$ (c) images of the leg ulcer and surrounding area. Selected regions of interest are indicated by white squares and marked as ROI 1, ROI 2, and ROI 3. 
Figure 2 shows a comparison of temperature and blood flow averaged for each ROI $(n=10)$, as well as spatial profiles extracted from the IR and LSCI time-averaged images $(n=10)$. As seen in Figure $2 a$, ROI 1 reported significantly lower temperatures compared to both ROIs 2 and 3. There was no statistical difference in temperature between ROIs 2 and 3. The spatial profiles of average temperature obtained with IR (Figure $2 \mathrm{~b}$ ) support these results, showing minimum temperature at ROI 1, maximum temperature in ROI 2, and slight temperature attenuation when approaching ROI 3. Blood flow in ROIs 1 and 2 was significantly higher when compared to the distant ROI 3, as seen in Figure 2c. There was no statistical difference in blood flow between ROIs 1 and 2. These results were supported by the spatial profiles of average blood flow obtained with LSCI (Figure 2d), which showed maximum blood flow in ROI 1, slightly lower blood flow at the ulcer boundary into ROI 2, and substantially decreasing blood flow toward ROI 3.

\section{IR: Temperature}

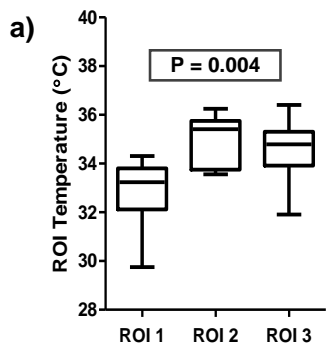

b)

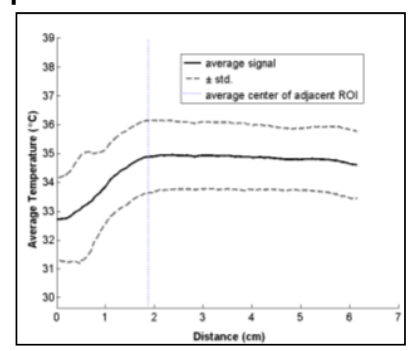

c)

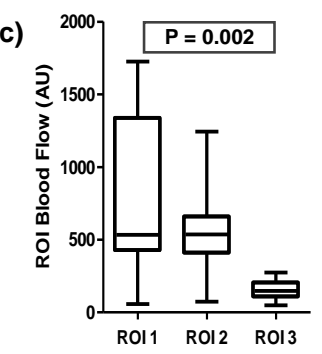

LSCI: Blood Flow

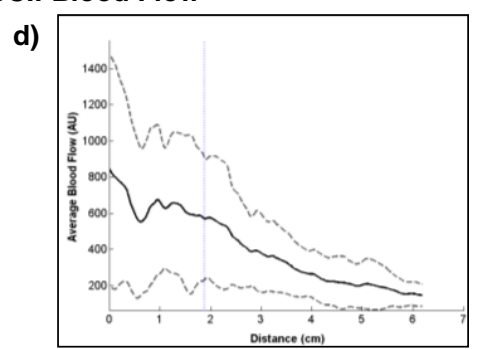

Fig. 2. ROI temperature (a) and blood flow (c) values are reported as medians across patients. $P$ values were calculated using a Kruskal-Wallis One-way ANOVA on ranks test to compare median values between ROIs. Corresponding spatial profiles extracted from IR (b) and LSCI (d) time-averaged images were averaged across patients and reported as average \pm standard deviation. Profiles were traced from the center of the ulcer (ROI 1, distance zero), through the center of $R O I$ 2, to the center of distant ROI 3. The vertical dotted blue line represents the average location of ROI $2(n=10)$.

The statistical comparisons between each ROI are summarized in Table 1.

Table 1. Comparison of temperature and blood flow averaged for each $R O I(n=10)$. $P$ values were calculated using a Wilcoxon signed rank test (paired) to compare median $R O I$ values across patients.

\begin{tabular}{|c|c|c|c|c|c|c|}
\hline Parameters & \multicolumn{3}{|c|}{ Average Temperature } & \multicolumn{3}{c|}{ Average Blood Flow } \\
\hline ROls & 1 vs. 2 & 1 vs. 3 & 2 vs. 3 & 1 vs. 2 & 1 vs. 3 & 2 vs. 3 \\
\hline Wilcoxon signed rank & $\mathrm{P}=0.002$ & $\mathrm{P}=0.002$ & $\mathrm{P}=0.131$ & $\mathrm{P}=0.322$ & $\mathrm{P}=0.010$ & $\mathrm{P}=0.002$ \\
\hline
\end{tabular}

Figure 3 shows the power density of temperature and blood flow oscillations computed for the individual frequency ranges of interest corresponding to endothelium-derived hyperpolarizing factor, rate of endothelial release of nitric oxide, sympathetic activity, and myogenic activity. Figure 3a shows temperature oscillations in each range for each $\mathrm{ROI}$, with the ulcer region (ROI 1) reporting significantly higher power in all ranges. Figure $3 \mathrm{~b}$ shows blood flow oscillations in each range for each ROI. Blood flow oscillations in ROIs 1 and 2 were significantly higher than in ROI 3, which displayed the lowest power density in all four ranges.

The power distribution in each ROI was also assessed by using power ratios, where each sub-range was normalized to the total power density in the $0.005-0.20 \mathrm{~Hz}$ range. There were no significant differences between ROIs in the distribution of temperature oscillations, with power appearing equally distributed across each range. In terms of blood flow oscillations, ROI 2 showed a significantly higher distribution of power in the NO-dependent endothelial range compared to ROI $3(\mathrm{p}<0.05)$. In all IR and LSCI measurements, oscillations were significantly higher in the wound compared to the distant areas in all three oscillation ranges.
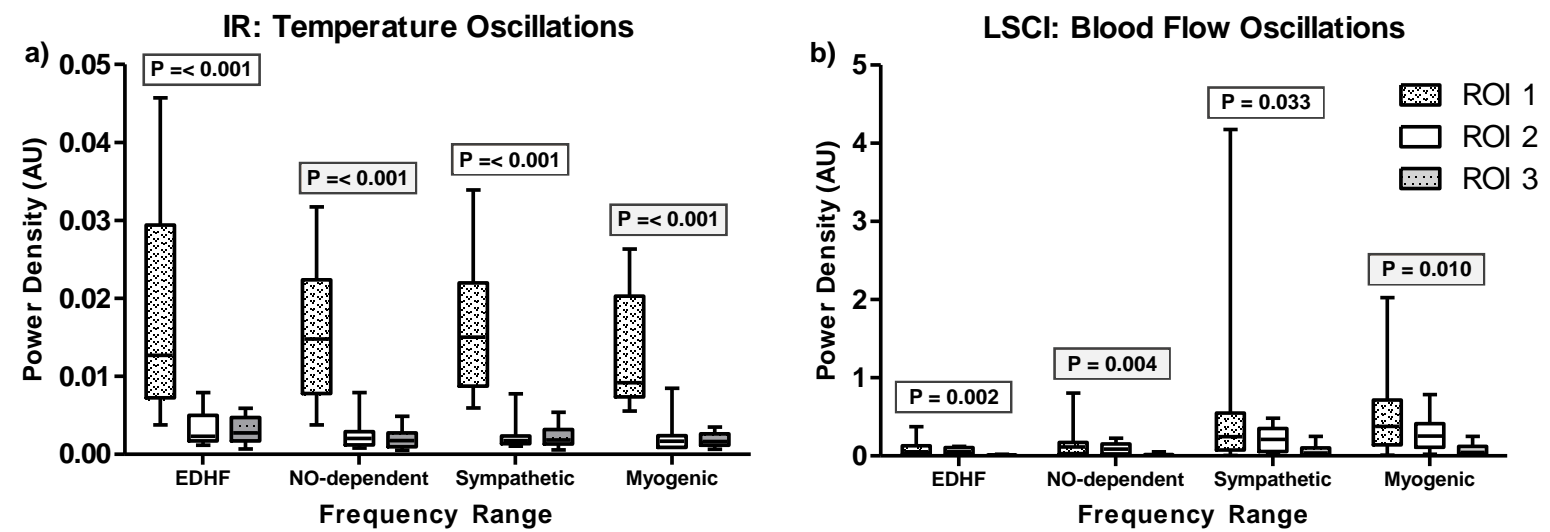

Fig. 3. Power densities of temperature (a) and blood flow (b) oscillations in the individual frequency ranges of interest for each ROI $(n=10)$. $P$ values were calculated using a Kruskal-Wallis One-way ANOVA on ranks test to compare median power density between ROls. 
The statistical comparison of each ROI in all frequency ranges is shown in Table 2. The significant differences in power density between ROls remained true when comparing total power density in the $0.005-0.20 \mathrm{~Hz}$ range $(p<0.05)$.

Table 2. Comparison of temperature and blood flow oscillations in individual frequency ranges of interest for each ROI $(n=10)$. $P$ values were calculated using a Wilcoxon signed rank test (paired) to compare median ROI power density across patients.

\begin{tabular}{|c|c|c|c|c|c|c|c|c|c|c|c|c|}
\hline Frequency Range & \multicolumn{3}{|c|}{$0.005-0.0095 \mathrm{~Hz}$} & \multicolumn{3}{|c|}{$0.0095-0.021 \mathrm{~Hz}$} & \multicolumn{3}{|c|}{$0.021-0.06 \mathrm{~Hz}$} & \multicolumn{3}{|c|}{$0.06-0.20 \mathrm{~Hz}$} \\
\hline ROIs & 1 vs. 2 & 1 vs. 3 & 2 vs. 3 & 1 vs. 2 & 1 vs. 3 & 2 vs. 3 & 1 vs. 2 & 1 vs. 3 & 2 vs. 3 & 1 vs. 2 & 1 vs. 3 & 2 vs. 3 \\
\hline Temperature & $\begin{array}{c}P= \\
0.002\end{array}$ & $\begin{array}{c}P= \\
0.004\end{array}$ & $\begin{array}{c}P= \\
1.000\end{array}$ & $\begin{array}{c}P= \\
0.002\end{array}$ & $\begin{array}{c}P= \\
0.002\end{array}$ & $\begin{array}{c}P= \\
0.432\end{array}$ & $\begin{array}{c}P= \\
0.002\end{array}$ & $\begin{array}{c}P= \\
0.002\end{array}$ & $\begin{array}{c}\mathrm{P}= \\
0922\end{array}$ & $\mathrm{P}=$ & $P=$ & $\begin{array}{c}P= \\
0922\end{array}$ \\
\hline Blood flow & $\begin{array}{c}P= \\
0.375\end{array}$ & $\begin{array}{c}P= \\
0.010\end{array}$ & $\begin{array}{c}P= \\
0.002\end{array}$ & $\begin{array}{c}P= \\
0.625\end{array}$ & $\begin{array}{c}P= \\
0.010\end{array}$ & $\begin{array}{c}P= \\
0.002\end{array}$ & $\begin{array}{c}\mathrm{P}= \\
0.432\end{array}$ & $\begin{array}{c}P= \\
0.037\end{array}$ & $\begin{array}{c}P= \\
0.006\end{array}$ & $\begin{array}{c}P= \\
0.492\end{array}$ & $\begin{array}{c}P= \\
0.010\end{array}$ & $\begin{array}{c}P= \\
0.002\end{array}$ \\
\hline
\end{tabular}

Figure $4 \mathrm{a}$ shows the calculated temperature oscillation image, where each pixel represents power density of temperature oscillations in the entire $0.005-0.20 \mathrm{~Hz}$ range. Temperature oscillations were visibly higher within the ulcer compared to surrounding and distant tissue. Some regions of increased power, however, were evident in the periwound tissue outside the chosen ROls. The spatial profile of temperature oscillations (Figure 4b) showed maximum power near $\mathrm{ROI} 1$, a dramatic decrease in power in ROI 2, and attenuation of power approaching ROI 3. Figure 4c shows the calculated blood flow oscillation image, with the highest power density of blood flow oscillations localized within the ulcer. The periwound tissue also showed increased power of blood flow oscillations compared to distant tissue. The spatial profile of blood flow oscillations (Figure 4d) showed maximum power in ROI 1, a small resurgence of power in ROI 2, and diminished power in ROI 3.
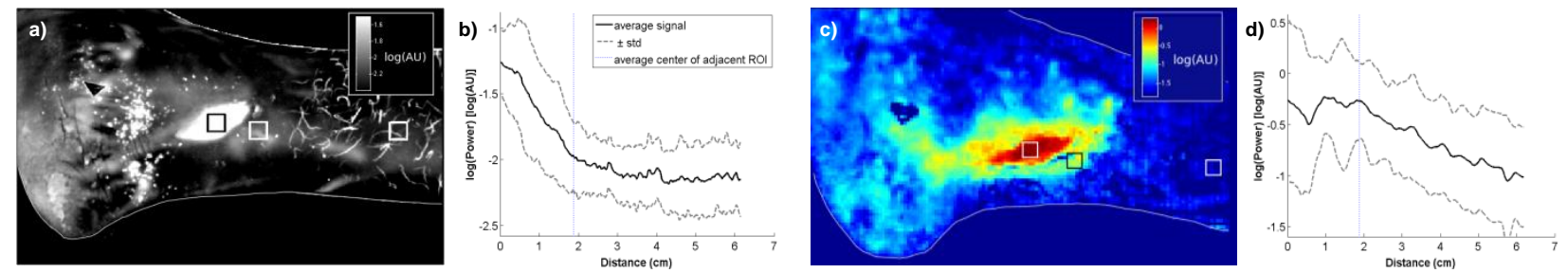

Fig. 4. Representative IR (a) and LSCI (c) oscillation images (power density in each pixel) for the $0.005-0.20 \mathrm{~Hz}$ range, with corresponding temperature (b) and blood flow (d) oscillation spatial profiles. Oscillation images are of a representative subject, while spatial profiles are averaged across patients and reported as average \pm standard deviation $(n=10)$. Note: images and profiles are in log scale for ease of interpretation.

To search for functional connectivity between different oscillatory frequencies in the ulcer and surrounding tissue, wavelet phase coherence plots were calculated for each pair of ROls for one subject (Figure 5). The white Gaussian noise threshold was set to one standard deviation above a pre-generated white noise reference curve and is represented by a thin black line in the Figure 5 plots. ROIs were considered to have significant coherence when their respective WPC values were above this white noise threshold. Each frequency range was analyzed by examining the average WPC in each range and comparing these values to the average white noise threshold in said range.

ROI 1 displayed significant coherence of temperature oscillations with ROI 2 in all four frequency ranges of interest (Figure 5a). ROIs 1 and 3 showed significant coherence of temperature oscillations in the EDHF and sympathetic ranges, while ROIs 2 and 3 had significant coherence in the sympathetic and myogenic ranges (Figure 5a). Coherent blood flow oscillations were observed between all ROIs in the NO-dependent and myogenic frequencies (Figure 5b). ROI 1 was also significantly coherent with ROIs 2 and 3 in the sympathetic range. No coherent blood flow oscillations were observed in the EDHF range.

a)

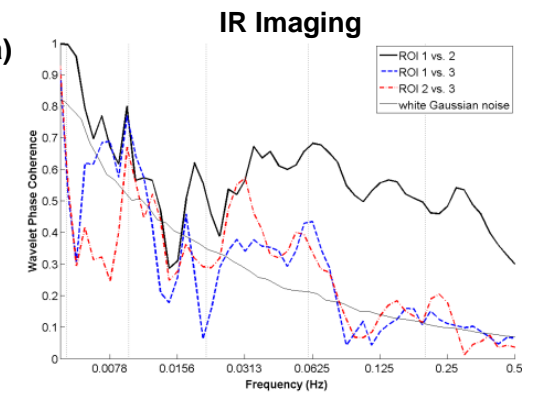

b)

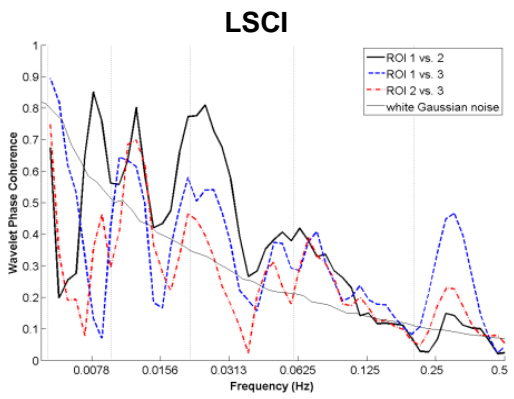

Fig. 5. Temperature (a) and blood flow (b) wavelet phase coherence plots showing coherence of oscillations between ROIs $(n=1)$. The line for white Gaussian noise represents one standard deviation above the white noise coherence curve (i.e. significant coherence). Bounds for the frequency ranges of interest are indicated by vertical dotted lines. Note: the frequency axis is in logarithmic scale. 
Table 3 shows the average WPC between all ROIs per frequency range, with the average white noise thresholds shown for reference.

Table 3. Average wavelet phase coherence of temperature and blood flow oscillations between ROls for each frequency range of interest $(n=1)$. The average white noise threshold for significant coherence in each range is shown for reference. Asterisks indicate phase coherence greater than the threshold.

\begin{tabular}{|c|c|c|c|c|c|c|c|c|}
\hline \multirow[b]{2}{*}{ WPC Comparison } & \multicolumn{4}{|c|}{ Temperature } & \multicolumn{4}{|c|}{ Blood Flow } \\
\hline & $\begin{array}{c}0.005- \\
0.0095 \mathrm{~Hz}\end{array}$ & $\begin{array}{l}0.0095- \\
0.021 \mathrm{~Hz}\end{array}$ & $\begin{array}{l}0.021- \\
0.06 \mathrm{~Hz}\end{array}$ & $\begin{array}{c}0.06- \\
0.20 \mathrm{~Hz}\end{array}$ & $\begin{array}{c}0.005- \\
0.0095 \mathrm{~Hz}\end{array}$ & $\begin{array}{l}0.0095- \\
0.021 \mathrm{~Hz}\end{array}$ & $\begin{array}{l}0.021- \\
0.06 \mathrm{~Hz}\end{array}$ & $\begin{array}{c}0.06- \\
0.20 \mathrm{~Hz}\end{array}$ \\
\hline ROls 1 vs. 2 & $0.756^{\star}$ & $0.489^{*}$ & $0.596^{\star}$ & $0.552^{\star}$ & 0.612 & $0.599^{*}$ & $0.461^{\star}$ & $0.188^{\star}$ \\
\hline ROls 1 vs. 3 & $0.655^{\star}$ & 0.286 & $0.346^{*}$ & 0.143 & 0.485 & $0.438^{*}$ & $0.338^{*}$ & $0.200^{*}$ \\
\hline ROls 2 vs. 3 & 0.469 & 0.351 & $0.401^{\star}$ & $0.153^{\star}$ & 0.398 & $0.437^{\star}$ & 0.233 & $0.175^{\star}$ \\
\hline White Noise Threshold & 0.631 & 0.408 & 0.255 & 0.145 & 0.631 & 0.408 & 0.255 & 0.145 \\
\hline
\end{tabular}

To identify areas that have high coherence of temperature oscillations with the ulcer, IR-derived wavelet phase coherence images were calculated for each frequency range of interest (Figure 6a-6d). These images compare the temperature oscillations of each pixel to the oscillations of average temperature within the ulcer by wavelet phase coherence analysis, where each pixel value is the average WPC calculated across the corresponding frequency range for that pixel. The generated image is a map showing the degree of coherence between the ulcer and the rest of the leg. Figure 6e-6f show images which contain pixels above the average white noise threshold (i.e. regions of significant coherence) for each range. As seen in the figure, IR imaging was able to demonstrate various regions that significantly cohered with oscillations of average temperature within the ulcer. These regions also varied depending on the frequency ranged analyzed.
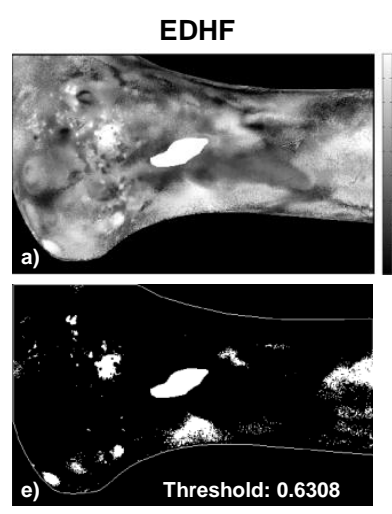

NO-dependent
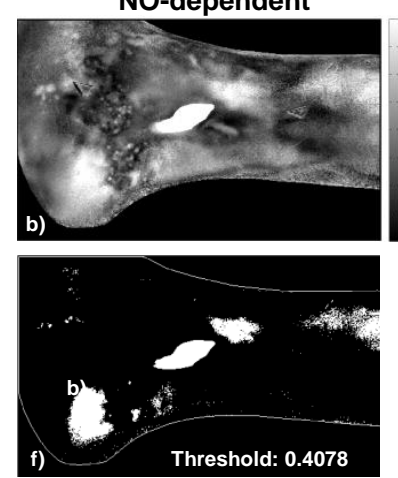

Sympathetic
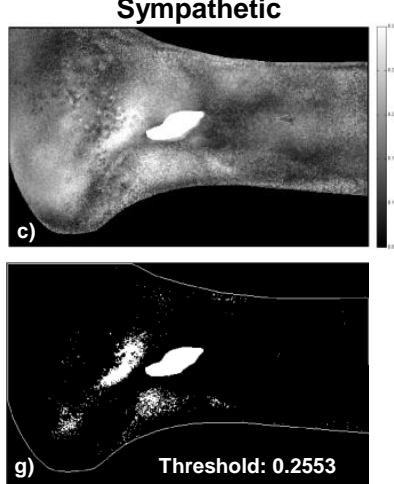

Myogenic
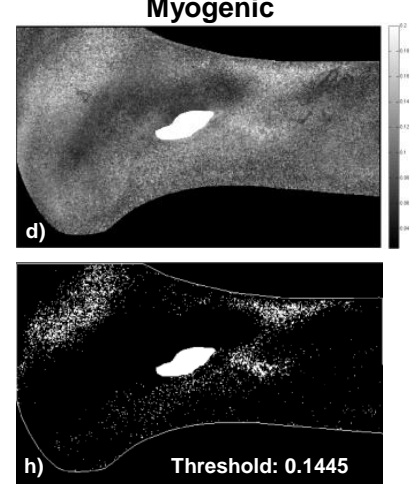

Fig. 6. IR-derived wavelet phase coherence and threshold images calculated for the frequency ranges $0.005-0.0095$ $\mathrm{Hz}(a, e), 0.0095-0.021 \mathrm{~Hz}(b, f), 0.021-0.06 \mathrm{~Hz}(c, g)$, and $0.06-0.20 \mathrm{~Hz}(d, h)$. These images compare oscillations of each pixel to the oscillations of average temperature within the ulcer by wavelet phase coherence analysis. Average white noise thresholds are shown for reference. Note: this analysis was performed for a single patient $(n=1)$.

Figure 7a-7d show the LSCI-derived wavelet phase coherence images, which compare blood flow oscillations of each pixel to the oscillations of average blood flow within the ulcer for each frequency range. As seen in the figure, LSCIderived WPC images did not show well-defined areas that cohered significantly with ulcer blood flow oscillations. The location and density of areas of significant coherence did not display a clear pattern similar to those seen in the IRderived WPC images (data not shown).
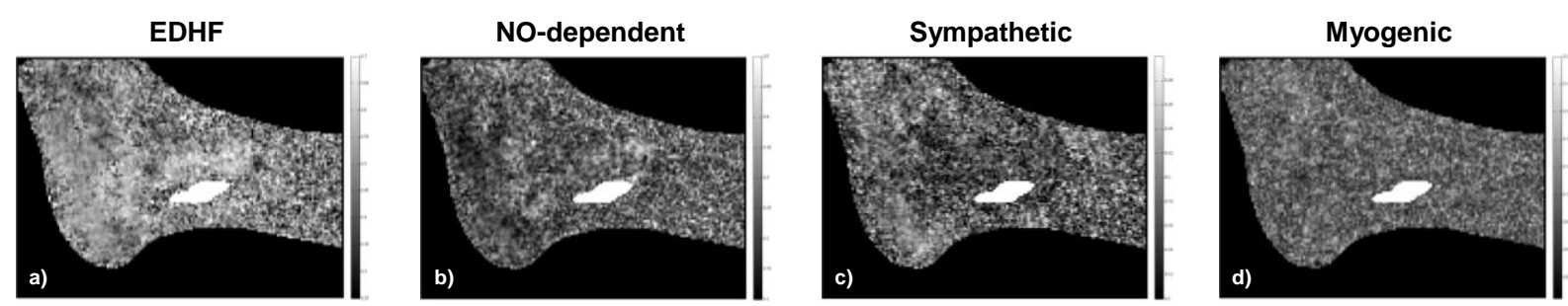

Fig. 7. LSCl-derived wavelet phase coherence and threshold images calculated for the frequency ranges $0.005-0.0095$ $\mathrm{Hz}$ (a), $0.0095-0.021 \mathrm{~Hz}$ (b), $0.021-0.06 \mathrm{~Hz}$ (c), and $0.06-0.20 \mathrm{~Hz}$ (d). These images compare oscillations of each pixel to the oscillations of average blood flow within the ulcer by wavelet phase coherence analysis. Note: this analysis was performed for a single patient $(n=1)$. 


\section{Discussion}

Simultaneous recordings of images were made of temperature and red blood cell flux in ulcers and surrounding tissues of patients with sickle cell anemia in order to assess spatial, temporal, and spectral aspects of vascular perfusion. Examination of the captured images revealed distinct distributions of temperature and blood flow related to ulceration, as seen in Figure $1 \mathrm{~b}-1 \mathrm{c}$.

Flow and temperature values were assessed at three selected ROls representing 1) the ulcer, 2) adjacent tissues, and 3) distant tissues. Analysis of these three ROls revealed significant differences among them in temperature and blood flow - and the respective oscillations of these variables. In IR imaging, ROI 1 displayed significantly lower temperature than ROIs 2 and 3, most likely due to evaporative cooling of the non-epithelialized ulcer surface. Spatial profiles of average temperature reinforced the distinction between the ulcer and the surrounding tissue, showing a dramatic increase in temperature at the transition from the ulcer to non-ulcerated tissue. ROI 1 also showed significantly higher temperature oscillations than the two other ROIs for all frequency ranges. The power of the oscillations within the ulcers was significantly higher than in the surrounding tissues for all frequency ranges analyzed. These frequency ranges have been associated in the literature with specific underlying mechanisms (EDHF, endothelial release of NO, sympathetic activity, and myogenic activity), all of which may play a role. Evaporative cooling of the ulcer surface may have affected the oscillations as well. The temperature oscillation images and their corresponding spatial profiles further illustrated the distinction between the ulcers and surrounding tissue, showing a sharp decrease in locally regulated temperature oscillation (at $0.005-0.20 \mathrm{~Hz}$ ) outside the ulcer border (Figure $4 \mathrm{a}-4 \mathrm{~b}$ ). Thus, IR imaging demonstrated temperature fluctuations characteristic of ulcer tissue and may be a suitable technique for quantitative assessment of ulceration.

Although the magnitude of temperature oscillations decreased rapidly away from the ulcer, wavelet phase coherence analysis of the three ROIs (Figure 5a) for one subject showed that these regions maintained significant coherence of oscillatory activity in the frequency ranges of interest, with the ulcer and periwound (rather than the distant region) displaying the strongest coherence. This suggests that, although these regions displayed different oscillatory amplitudes, the ulcer and periwound oscillations may share a regulatory mechanism. Distinct regions outside of the ulcer had significant wavelet phase coherence with the ulcer's temperature oscillations that were readily visualized in the WPC images from one patient (Figure $6 \mathrm{e}-6 \mathrm{~g}$ ). These images showed that changes in ulcer temperature, most likely indicating blood flow, coincided with other regions of the skin. This implies coordination of the microcirculation between the ulcers and surrounding areas, possibly by the conductive vasodilation mechanism mentioned earlier [7]. This coherence between ulcer and non-ulcer tissue should be further explored to determine whether these regions have predictive value during wound assessment, as observation of these regions may lead to specific markers to ulcer precursors or ulcer healing.

LSCI detected significantly higher blood flow in the ulcer and periwound region than in the distant control region. Increased blood flow localized to the ulcer may suggest a higher degree of vascular recruitment in this area, potentially related to wound healing. The increased red blood cell flux measured directly by LSCI could represent a compensatory response to nearby blood vessel occlusion by rigid red cells [14]. Spatial profiles for blood flow (Figure 2d) show decreased perfusion with increased distance from the ulcer. Blood flow oscillations were also significantly higher in the ulcer and periwound regions in all four frequency ranges, suggesting increased EDHF, NO-dependent, sympathetic, and myogenic activity in these regions. The blood flow oscillation images and their corresponding spatial profiles showed decreased activity of $0.005-0.20 \mathrm{~Hz}$ oscillations with increasing distance from the ulcer (Figure 4d). Whether the increased perfusion and oscillatory activity in the ulcer and adjacent regions were the result of a compensatory healing mechanism or of previous ulceration (e.g. altered microvasculature in fibrous scar tissue), or whether the alterations could even have promoted the ulcer, remains unclear.

WPC analysis of blood flow oscillations in the three ROls revealed instances of significant coherence in all frequency ranges, except the EDHF range, for one subject (Figure 5b). Coherent blood flow oscillations were observed between all ROIs in the NO-dependent and myogenic frequencies, while only the ulcer was significantly coherent with periwound and distant regions in the frequency range associated with sympathetic activity. In each case, the periwound and distant tissues displayed the least coherence. Coherence between regions suggests the existence of a communicating network of vascular oscillators. The WPC images, however, did not reveal well-defined regions of significant coherence between the ulcer and its surrounding tissue (Figure 7). Although these images showed that blood flow changes in the ulcer were somewhat reflected in other areas of the skin, the regions that were above the white noise threshold did not display a common pattern, which would suggest a communicative vascular network (data not shown). Further studies of blood flow oscillations in and around the ulcer are required to determine possible patterns of coherence.

The discrepancies between the results for IR imaging and LSCI might be explained by the inherent differences in the techniques. IR imaging is an indirect measure of blood flow through analysis of temperature. Although thermal images are sensitive to blood flow, other sources of heat (e.g., metabolism) influence temperature [15]. LSCl, however, is a direct measure of blood flow through analysis of red blood cell flux and, therefore, has an assumed specificity of $100 \%$ for blood flow measurements. Also, IR imaging is sensitive to temperature changes at a depth of about $2 \mathrm{~cm}$ beneath the skin [16], and as such may reveal a signature of ulcer development and healing within the skin, as well at the skin's surface. $\mathrm{LSCl}$, on the other hand, detects microperfusion only to a depth of about $0.3 \mathrm{~mm}$ and is more specific to nutritive perfusion [17, 18]. Additionally, each imaging technique has a different sensitivity to the observed frequencies of oscillation. IR imaging is more suitable for measurement of low-frequency temperature fluctuations due to the damping 
effect of the skin, which causes the amplitude of heat fluctuations to decay as an exponential function of frequency [2]. Oscillations of red blood cell flux, as detected by LSCI, however, are not subject to this effect, and higher frequency information is assumed to be preserved [18]. Thus, the discrepancies in the results seen by IR imaging and LSCI are likely attributable to their different physical bases of measurement, measuring depths, and sensitivities to the skin's damping effects on frequency. For these reasons, the results for each method reflect different aspects of blood flow and provide complementary information.

\section{Conclusion}

IR imaging demonstrated low-frequency temperature oscillations in ulcers that sharply distinguished the ulcers from surrounding tissues, whereas LSCl demonstrated alterations in microcirculatory perfusion that distinguished the ulcers from distant (control) tissues. The LSCI differences in microcirculation between ulcers and their immediately adjacent tissues were not, however, statistically significant. Wavelet phase coherence analysis of IR images was done for one patient and showed coherence between oscillations within the ulcer and some (but not all) regions outside the ulcer, suggesting a shared regulatory mechanism for the coherent regions. The imaging modalities discussed in this article are complementary and, when used together, may provide a means for passive, non-invasive, quantitative assessment of vascular tone and possible functional connectivity between ulcers and their surroundings. Further study of ulceration by both techniques may delineate specific pathways related to vascular recruitment and/or compensatory activity.

\section{Acknowledgements}

This research was supported by the Intramural Research Program of the National Institute of Biomedical Imaging and Bioengineering, and by the Division of Intramural Research of the National Heart, Lung, and Blood Institute at the National Institutes of Health. We would like to thank Dr. Henry Eden for his guidance in this work and for proofreading this manuscript. We acknowledge the expert protocol coordination by Marlene Peters-Lawrence and protocol management by Mary K. Hall. 


\section{REFERENCES}

[1] Minniti, C.P., Eckman, J., Sebastiani, P., Steinberg, M.H., and Ballas, S.K. Leg ulcers in sickle cell disease. American Journal of Hematology, vol. 85, pp. 831-833, 2010.

[2] Liu, W., Meyer, J., Scully, C.G., Elster, E., and Gorbach, A.M. Observing Temperature Fluctuations in Humans Using Infrared Imaging. QIRT 2010.

[3] Rege, A., Thakor, N.V., Rhie, K., and Pathak, A.P. In vivo laser speckle imaging reveals microvascular remodeling and hemodynamic changes during wound healing angiogenesis. Angiogenesis, vol. 15, pp. 87-98, 2012.

[4] moorFLPI Full-Field Laser Perfusion Image USER GUIDE. Moor Instruments Limited, Issue 2, 2007.

[5] Sheppard, L.W., Vuksanovic, V., McClintock, P.V.E., and Stefanovska, A. Oscillatory dynamics of vasoconstriction and vasodilation identified by time-localized phase coherence. Physics in Medicine and Biology, vol. 56, pp. 3583-3601, 2011.

[6] Segal, S. Regulation of Blood Flow in the Microcirculation. Microcirculation, vol. 12, pp. 33-45, 2005.

[7] Bagher, P., and Segal, S. S. Regulation of blood flow in the microcirculation: role of conducted vasodilation. Acta Physiologica, vol. 202, pp. 271-284, 2011.

[8] Ambrozy, E., Waczulikova, I., Willfort-Ehringer, A., Ehringer, H., Koppensteiner, R., and Gschwandtner, M.E. Microcirculation in mixed arterial/venous ulcers and the surrounding skin: Clinical study using a laser Doppler perfusion imager and capillary microscopy. Wound Repair and Regeneration, vol.17, pp. 19-24, 2009.

[9] Ambrozy, E., Waczulikova, I., Willfort, A., Bohler, K., Cauza, K., Ehringer, H., Heinz, G., Koppensteiner, R., Maric, S., and Gschwandtner, M.E. Healing process of venous ulcers: the role of microcirculation. International Wound Journal, 2012.

[10] Nakagami, G., Sanada, H., lizaka, S., Kadono, T., Higashino, T., Koyanagi, H., and Haga, N. Predicting delayed pressure ulcer healing using thermography: a prospective cohort study. Journal of Wound Care, vol. 19, pp. 465-472, 2010.

[11] Frackowiak, et al. (Eds.). "Human brain function," Academic Press (London), Ch2, 1997.

[12] Aboy, M., Crespo, C., McNames, J., Bassale, J, Jenkins, L., and Goldstein, B. A biomedical signal processing toolbox, In Proc. 16th International EURASIP Conference BIOSIGNAL 2002, Brno, Czech Republic, Vol. 16, 2628 June 2002, pp. 49-52.

[13] Bernjak, A., Cui, J., Iwase, S., Mano, T., Stefanovska, A., and Eckberg, D.L. Human sympathetic outflows to skin and muscle target organs fluctuate concordantly over a wide range of time-varying frequencies. Journal of Physiology, vol. 590, pp. 363-375, 2012.

[14] Rodgers, G.P., Schecter, A.N., Noguchi, C.T., Klein, H.G., Nienhuis, A.W., and Bonner R.F. Periodic Microcirculatory Flow in Patients with Sickle-Cell Disease. New England Journal of Medicine, vol. 311, pp. 1534-1538, 1984.

[15] Gorbach, A.M., Wang, H., Wiedenbeck, B., Liu, W., Smith, P.D., and Elster, E. Functional Assessment of Hand Vasculature Using Infrared and Laser Speckle Imaging. Proc. of SPIE, vol. 7169, 2009.

[16] Ivanitsky, G.R., Deev, A.A., Pashovkin, T.N., Khizhnyak, E.P., Khizhnyak, L.N., and Tsyganov, M.A. Display Peculiarities of Hypodermic Heating Sources on the Human Body Surface. Biochemistry, Biophysics, and Molecular Biology, vol. 420, pp. 130-134, 2008.

[17] Mahé, G., Humeau-Heurtier, A., Durand, S., Leftheriotis, G., and Abraham, P. Assessment of Skin Microvascular Function and Dysfunction With Laser Speckle Contrast Imaging. Circulation Cardiovascular Imaging, vol. 5, pp. 155-163, 2012.

[18] Roustit, M., and Cracowski, J.L. Non-invasive Assessment of Skin Microvascular Function in Humans: An Insight Into Methods. Microcirculation, vol. 19(1), pp. 47-64, 2012. 